\title{
Evaluating associations between the benefits and risks of drug therapy in type 2 diabetes: a joint modeling approach
}

This article was published in the following Dove Press journal:

Clinical Epidemiology

\author{
John M Dennis' \\ Beverley M Shields² \\ Angus $G$ Jones ${ }^{2}$ \\ Ewan R Pearson ${ }^{3}$ \\ Andrew T Hattersley ${ }^{2}$ \\ William E Henley' \\ On behalf of the MASTERMIND \\ consortium \\ 'Health Statistics Group, University \\ of Exeter Medical School, Exeter, \\ UK; ${ }^{2}$ National Institute for Health \\ Research Exeter Clinical Research \\ Facility, University of Exeter Medical \\ School, Exeter, UK; ${ }^{3}$ Division of \\ Molecular and Clinical Medicine, \\ Ninewells Hospital and Medical \\ School, University of Dundee, \\ Dundee, UK
}

The licence of this article has been updated from CC-BY-NC to CC-BY on March 12, 2019.
Correspondence: William E Henley Health Statistics Group, University of Exeter Medical School, South Cloisters, St Luke's Campus, Magdalen Road, Exeter EXI 2LU, UK

Tel+44I392726088

Email W.E.Henley@exeter.ac.uk
Objective: Precision medicine drug therapy seeks to maximize efficacy and minimize harm for individual patients. This will be difficult if drug response and side effects are positively associated, meaning that patients likely to respond best are at increased risk of side effects. We applied joint longitudinal-survival models to evaluate associations between drug response (longitudinal outcome) and the risk of side effects (survival outcome) for patients initiating type 2 diabetes therapy. Study design and setting: Participants were randomized to metformin (MFN), sulfonylurea (SU), or thiazolidinedione (TZD) therapy in the A Diabetes Outcome Progression Trial (ADOPT) drug efficacy trial $(n=4,351)$. Joint models were parameterized for 1$)$ current $\mathrm{HbA} 1 \mathrm{c}$ response (change from baseline in $\mathrm{HbA} 1 \mathrm{c}$ ) and 2) cumulative $\mathrm{HbA} 1 \mathrm{c}$ response (total HbA1c change).

Results: With MFN, greater HbA1c response did not increase the risk of gastrointestinal events (HR per 1\% absolute greater current response 0.82 [95\% CI 0.67, 1.01]; HR per 1\% higher cumulative response 0.90 [95\% CI 0.81, 1.00]). With SU, greater current response was associated with an increased risk of hypoglycemia (HR 1.41 [95\% CI 1.04, 1.91]). With TZD, greater response was associated with an increased risk of edema (current HR 1.45 [95\% CI 1.05, 2.01]; cumulative 1.22 [95\% CI 1.07, 1.38]) but not fracture.

Conclusion: Joint modeling provides a useful framework to evaluate the association between response to a drug and the risk of developing side effects. There may be great potential for widespread application of joint modeling to evaluate the risks and benefits of both new and established medications.

Keywords: diabetes mellitus, type 2, drug-related side effects, HbA1c, hypoglycemia, joint model, precision medicine, thiazolidinediones, metformin, sulfonylurea compounds, ADOPT, edema

\section{Plain language summary}

Purpose of study: An overlooked question in precision / stratified medicine and when evaluating new medications is: are the benefits and risks of a drug associated? Joint longitudinal-survival models can be applied to answer this question when, as in type 2 diabetes, drug response is measured by a longitudinal biomarker $(\mathrm{HbAlc})$ and risks of side-effects can be represented as a time-to-event outcome.

What did we do and find? We used joint longitudinal-survival models to show novel associations between the benefit of greater drug response and the risk of common side effects for three glucose-lowering medications for patients with type 2 diabetes. Greater drug response was associated with an increased risk of hypoglycemia with sulfonylureas and edema with thiazolidinediones. In contrast, there was no evidence of an increased risk of gastrointestinal side effects with metformin. 
What do the findings mean? Joint models provide a novel, flexible, and robust approach to study the associations between the risks and benefits of drug therapy. Precision/stratified medicine studies seeking to identify patients or subgroups likely to respond well to a drug should also evaluate whether the same patients are at increased risk of side effects.

\section{Introduction}

There is an increasing interest in applying a precision medicine approach to select the most appropriate drug for a patient or subgroup of patients, in order to either improve response or reduce side effects. ${ }^{1,2}$ An important but overlooked question, particularly if side effects are a result of the primary pharmacological effect of the drug, is whether the patients most likely to benefit are also at greatest risk of side effects. Type 2 diabetes is an ideal candidate for precision medicine, as there are many drug options to lower blood glucose (as measured by HbA1c), but each drug has a different mechanism of action and specific side effects. However, the association between $\mathrm{HbA1c}$ response and side effects is unknown for all drug options. If patients likely to have a greater $\mathrm{HbA} 1 \mathrm{c}$ response to a specific drug are also at increased risk of side effects, this may limit the clinical utility of any precision approach to type 2 diabetes therapy.

To date, no robust framework has been proposed to evaluate the association between drug response and risk of side effects. In type 2 diabetes, HbA1c is measured repeatedly over time (a longitudinal process), while side effect risk can be modeled as a time-to-event process. In this scenario, joint longitudinal-survival modeling is the preferred approach to evaluate the association between both processes. ${ }^{3-6}$ Joint models attempt to capture the true, unobserved, longitudinal trajectory (in reality, HbA1c is measured intermittently and is subjected to measurement error from random noise and biological variation). This means that joint models can reduce bias and improve efficiency compared with simpler approaches. ${ }^{5,7}$ Joint models have been applied in many diseases including recently in type 1 diabetes (autoantibodies and time to disease onset), ${ }^{8-11}$ but not to our knowledge in type 2 diabetes, or more broadly to evaluate the association between drug response and the risk of side effects.

In this study, we applied joint modeling to evaluate the association between drug response and the risk of established side effects for three widely used type 2 diabetes drugs and, thus, further evaluate the potential for precision drug therapy in type 2 diabetes.

\section{Methods}

\section{Overview}

Our aim was to understand whether the degree of glycemic response to three common glucose-lowering drugs altered the risk of developing a side effect. To answer this question, we examined the association between the following two outcomes: 1) HbA1c response (as measured by change from baseline in $\mathrm{HbA1c}$ ) and 2) risk of developing a side effect (gastrointestinal [GI] events, hypoglycemia, edema, and fracture).

\section{Setting and design}

We used individual participant level data from A Diabetes Outcome Progressing Trial (ADOPT) randomized trial, ${ }^{12}$ accessed through the Clinical Trial Data Transparency Portal under approval from GlaxoSmithKline (GSK) (Proposal-930). ${ }^{13}$ ADOPT was a prospective head-to-head drug trial including treatment-naive participants with type 2 diabetes who were randomized to metformin (MFN), the sulfonylurea (SU) glyburide, or the thiazolidinedione (TZD) rosiglitazone ( $n=4,351$ participants). The aim of ADOPT was to evaluate the long-term efficacy of TZD therapy compared to SU and MFN, and the primary outcome was time to therapy failure (confirmed fasting plasma glucose $\geq 180 \mathrm{mg} / \mathrm{dL}$ ). Study visits were every 2 months in year 1 , then every 3 months up to 5 years. Clinically determined adverse events were recorded at each study visit, including GI events, hypoglycemia, edema, and fracture. Biomarkers including $\mathrm{HbAlc}$ were recorded at each visit. ADOPT participants in the intention to treat population with a valid baseline $\mathrm{HbA1c}$ were eligible for our study. Participants were censored if they reached the trial primary endpoint of glycemic failure, trial-recorded study withdrawal, or at 5 years after starting therapy as in the ADOPT main analysis.

\section{Study outcomes}

Our time-to-event outcomes were the first occurrence of four established drug-specific side effects, over a 5-year period. For MFN, the outcome of interest was a GI event, for SU, it was hypoglycemia (patient self-reported), and for TZD, we evaluated edema and bone fractures. ${ }^{12}$ Each drug and side effect combination was analyzed separately. We excluded patients with a pre-trial history of edema from the edema analysis ( $6 \%$ of patients), but pre-trial hypoglycemia, GI, and fracture records were not available to do the same for other side effects. Due to the high number of GI events, we repeated the GI analysis restricted 
to only moderate/severe and severe events as sensitivity analysis. The longitudinal outcome of interest was $\mathrm{HbA} 1 \mathrm{c}$ response as measured by change from baseline in $\mathrm{HbA} 1 \mathrm{c}$ (HbA1c at each study visit [\%] - baseline HbA1c [\%]). Throughout HbA1c percentages refer to absolute values rather than percentage changes. To test the specificity of our findings, we repeated the analysis for each side effect for the other drugs.

\section{Statistical analysis}

We used a joint model with two parameterizations (Models 1 and 2) and two standard time-to-event models (Models 3 and 4), for comparison, to evaluate the association between $\mathrm{HbA} 1 \mathrm{c}$ response and the risk of developing a side effect. A fundamental difference between each model was in the method to estimate $\mathrm{HbA} 1 \mathrm{c}$ response, as illustrated in Figure 1. Each side effect was evaluated separately, and the same modeling approach was applied for each side effect. Participants were followed up for up to 5 years from randomization. As we were assessing the association between side effects and response, all participants required at least one pre-side effect $\mathrm{HbA} 1 \mathrm{c}$ measure (meaning that participants with very early side effects were excluded: $4 \%$ of participants with edema, $3 \%$ of participants with fracture, $20 \%$ of participants with hypoglycemia, and $12 \%$ of participants with GI events). All models were adjusted for baseline HbA1c. ${ }^{14}$ Model setups were as follows.

\section{Joint longitudinal-survival models}

We used a maximum likelihood joint longitudinal-survival model to simultaneously assess the association between $\mathrm{HbAlc}$ response (longitudinal process) and the risk of developing a side effect (survival process). The joint model consisted of the following two parts: a longitudinal submodel and a survival submodel linked through shared subject-specific random effects. ${ }^{6}$

In the general survival submodel, the hazard for patient $i\left(h_{i}(t)\right)$ can be represented as

$$
h_{i}(t)=h_{0}(t) \exp \left(w_{i}^{T} \gamma+\alpha m_{i}(t)\right)
$$

where $h_{0}(t)$ is the baseline hazard, $w_{i}$ are baseline covariates, $\gamma$ are regression coefficients, $m_{i}(t)$ is the "true, unobserved" longitudinal biomarker (estimated from the longitudinal submodel), and $\alpha$ quantifies the association between the longitudinal biomarker and the time-to-event process. ${ }^{6}$

We derived $m_{i}(t)$ from the observed $\mathrm{HbAl} \mathrm{c}$ response data using a linear mixed effects model with a nonlinear term for time (as $\mathrm{HbA} 1 \mathrm{c}$ response is typically nonlinear):

$$
y_{i}(t)=m_{i}(t)+\varepsilon_{i}(t)
$$

$=\beta_{0}+\beta_{1} N\left(t_{i}\right)_{1}+\beta_{2} N\left(t_{i}\right)_{2}+\beta_{3}$ Baseline HbA1c $+b_{i 0}+b_{i 1} N\left(t_{i}\right)_{1}$ $+b_{i 2} N\left(t_{i}\right)_{2}+\varepsilon_{i}(t)$

where $y_{i}$ is the observed $\mathrm{HbA} 1 \mathrm{c}$ change from baseline and $m_{i}$ is the "true", unobserved HbAlc change from

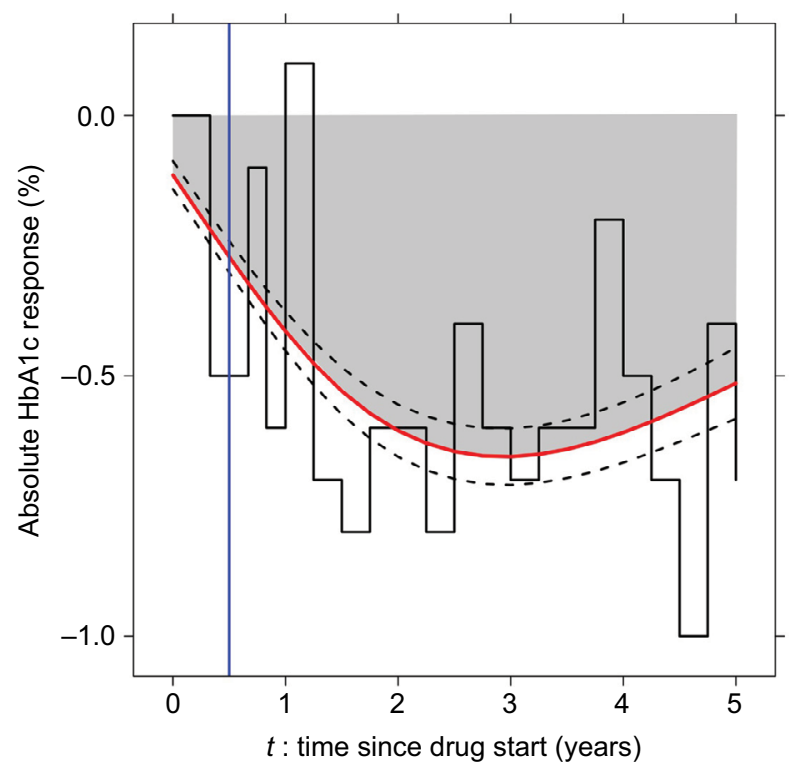

Figure I Approaches to estimating HbAlc (\%) response.

Notes: Model I: estimate current $\mathrm{HbAlc}$ response using a joint model (red line with black dotted $95 \% \mathrm{Cls}$ ). Model 2: estimate cumulative $\mathrm{HbAlc}$ response using a joint model (gray-shaded area). Model 3: carry forward the most recently observed value of HbAlc response until the next measurement (LOCF approach, black step function). Model 4: take the observed $\mathrm{HbAlc}$ response at a single time point of 6 months (blue line).

Abbreviation: LOCF, last observation carried forward. 
baseline. $N\left(t_{i}\right)_{1}$ and $N\left(t_{i}\right)_{2}$ denote the basis for a nonlinear natural cubic spline of time with one internal knot at the 50th percentile of follow-up time (included in both the fixed and random effect parts of the longitudinal $\mathrm{HbA} 1 \mathrm{c}$ submodel), $b_{i}$ is a vector of subject-specific random effects, $b_{i} \sim N(\mathrm{O}, \widetilde{\mathrm{D}})$ where $\widetilde{\mathrm{D}}$ is the unstructured covariance matrix of random effects, $\varepsilon_{i}$ is the vector of residuals, and $\varepsilon_{i} \sim N\left(\mathrm{O}, \sigma^{2}\right)$, where $\sigma^{2}$ is the covariance matrix of the residuals. ${ }^{6}$ For models of hypoglycemia with MFN and edema with SUs, we used a linear term for the random effect of time to achieve model convergence.

Model 1: joint model current value (JMcv). To assess the association between the current value of $\mathrm{HbA} 1 \mathrm{c}$ response and the risk of side effects (the standard formulation of the joint model), we incorporated $m_{i}$ from the longitudinal submodel as a time-dependent covariate in the survival submodel:

$$
h_{i}(t)=h_{0}(t) \exp \left\{\gamma_{0} \text { Baseline HbA1c }+\alpha m_{i}(t)\right\}
$$

Model 2: joint model cumulative HbAlc (JMcum). To evaluate whether the risk of side effects was associated with total rather than current $\mathrm{HbA} 1 \mathrm{c}$ response, we specified a second formulation of the joint model to assess the association between cumulative $\mathrm{HbA} 1 \mathrm{c}$ response (total $\mathrm{HbA} 1 \mathrm{c}$ response estimated as area under the curve) and the risk of side effects, by including $\int_{0}^{t} m_{i}(s) d s$, the integral of the longitudinal $\mathrm{HbA} 1 \mathrm{c}$ response trajectory up to time $t$, in the time-to-event submodel:6,15

$$
h_{i}(t)=h_{0}(t) \exp \left\{\gamma_{0} \text { Baseline HbA1c }+\alpha \int_{0}^{t} m_{i}(s) d s\right\}
$$

For Models 1 and 2, we used a B-spline with five internal knots to flexibly model the baseline hazard function. We examined the fit of submodels using residual plots. Models 1 and 2 were fitted using the JM package in R. ${ }^{16}$

Model 3: last observation carried forward (LOCF) analysis. We included observed $\mathrm{HbA} 1 \mathrm{c}$ response $(\mathrm{HbA} 1 \mathrm{c}$ at time $t$-baseline $\mathrm{HbA} 1 \mathrm{c}$ ) as a time-dependent covariate in a Cox proportional hazards model. This approach does not correct for measurement error and assumes that $\mathrm{HbA} 1 \mathrm{c}$ response is constant between measurements. HRs represent the increased risk of a side effect for a 1-unit (\%) absolute increase in the most recent value of $\mathrm{HbA} 1 \mathrm{c}$ change from baseline at time $t$.

Model 4: single estimate of HbAlc response at 6 months $(6 m R)$. We evaluated the association between HbAlc response at 6 months and the subsequent risk of developing a side-effect. In this two-stage approach, we first estimated a single estimate of $\mathrm{HbA} 1 \mathrm{c}$ response as a change score at 6 months. In the second stage, we used this estimate as the exposure in a Cox hazards survival model with delayed entry to 6 months. Participants who developed a side effect prior to 6 months or had no $\mathrm{HbA} 1 \mathrm{c}$ record at 6 months were excluded from this analysis.

\section{Results}

The most common side effects were GI side effects with MFN (37\%), followed by hypoglycemia with SU therapy (26\%). TZD side effects were less common (edema 13\% and fracture $7 \%$; Table 1 ). The median follow-up was greater than 2.5 years in each cohort (for other participant characteristics, refer Table S1). Each side effect occurred more frequently on these therapies than on the comparator drugs (Table S2).

\section{Joint model associations between $\mathrm{HbAlc}$ response and risk of side effects Gl events}

With MFN, we found consistent evidence for an association between greater $\mathrm{HbA} 1 \mathrm{c}$ response and reduced risk of a GI side effect (Figure 2A). We observed a similar association for moderate/severe GI events (20\% of patients) and no association for severe GI events (3\% of patients) (Table S3). We found no evidence of an association with TZDs and SUs (Tables 2 and $\underline{\mathrm{S} 3}$ ).

\section{Hypoglycemia}

With SUs, we found that greater current $\mathrm{HbA1c}$ response was associated with an increased risk of hypoglycemia

\begin{tabular}{|c|c|c|c|c|}
\hline & Metformin - GI & SU - hypo & TZD - edema & TZD - fracture \\
\hline Number of participants & 1,200 & 1,052 & $|, 24|$ & $|, 3| \mathrm{I}$ \\
\hline Number of events (\%) & $440(37 \%)$ & $270(26 \%)$ & $164(13 \%)$ & $88(7 \%)$ \\
\hline Baseline HbAlc (\%) & $7.3(6.7 ; 7.9)$ & $7.3(6.7 ; 7.9)$ & $7.3(6.7 ; 7.9)$ & $7.3(6.7 ; 7.9)$ \\
\hline Number of recorded $\mathrm{HbAlc}$ & $13(6 ; 19)$ & $12(5 ; 19)$ & $18(9 ; 20)$ & $18(10 ; 21)$ \\
\hline Study follow-up (years) & $2.8(1.0 ; 4.2)$ & $2.5(0.9 ; 4.2)$ & $4.0(1.8 ; 4.7)$ & $4.0(2.1 ; 4.7)$ \\
\hline
\end{tabular}

Table I Participant numbers and study follow-up for each primary drug: side effect cohort (Models I-3)

Note: Data are median (IQR) unless stated (refer Table S4 for participants included in Model 4).

Abbreviations: GI, gastrointestinal; SU, sulfonylurea; TZD, thiazolidinedione. 
A

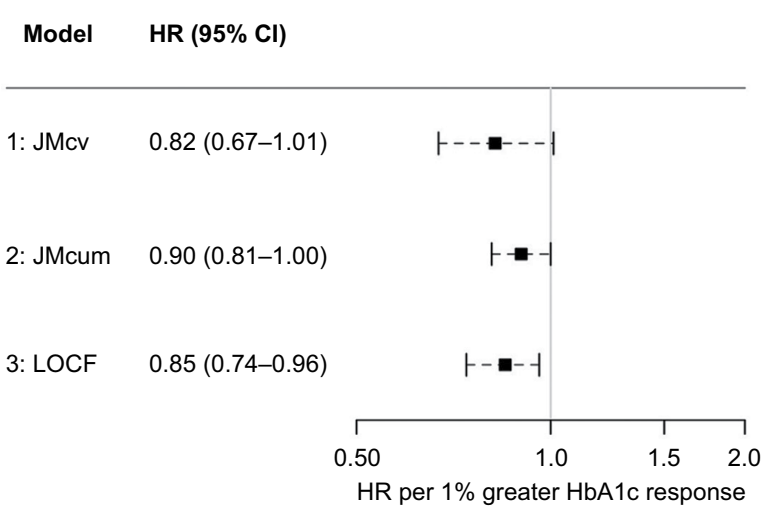

C

Model HR $(95 \% \mathrm{Cl})$

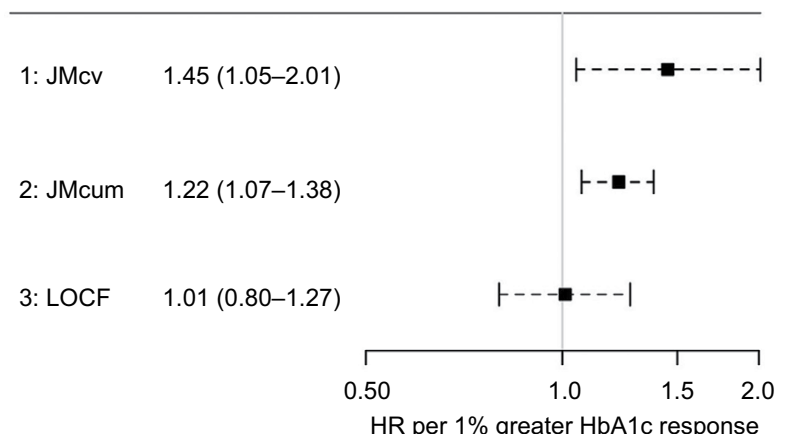

B

SU - hypoglycemia

Model HR $(95 \% \mathrm{Cl})$

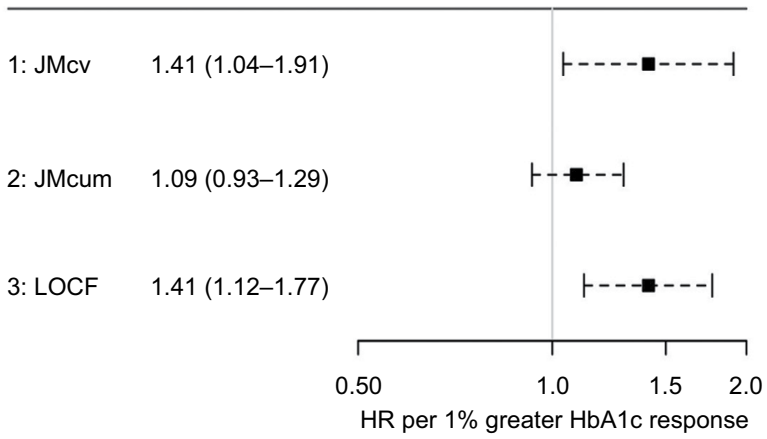

D

TZD - fracture

Model HR $(95 \% \mathrm{Cl})$

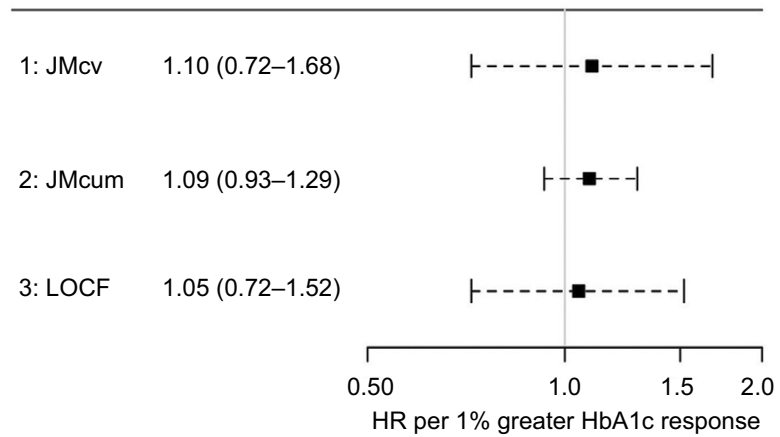

Figure 2 HRs for the association between $\mathrm{HbAlc}$ response and the risk of a drug-specific side effect (models I-3).

Notes: HRs $(95 \% \mathrm{Cl})$ represent the increase in the risk of side effect for a $1 \%$ greater absolute HbAlc response. A HR of greater than I indicates an increased risk of side effect with greater $\mathrm{HbAlc}$ response.

Abbreviations: JMcum, joint model cumulative HbAIc; JMcv, joint model current value; LOCF, last observation carried forward; MFN, metformin; SU, sulfonylurea; TZD, thiazolidinedione.

(Model 1: JMcv; Figure 2B). We found no evidence for an association between the risk of hypoglycemia and cumulative $\mathrm{HbA1c}$ response (Model 2: JMcum). With TZD therapy, although the absolute risk of hypoglycemia was much lower than with SU therapy (8 vs $26 \%$ ), greater current and cumulative $\mathrm{HbA} 1 \mathrm{c}$ responses were associated with an increased risk of hypoglycemia. There was no evidence of an association between response and hypoglycemia with MFN (Table 2).

\section{Edema}

With TZDs, greater current (Model 1: JMcv) and cumulative (Model 2: JMcum) HbA1c responses were associated with an increased risk of edema (Figure 2C). We found no evidence of an association between $\mathrm{HbAl} \mathrm{c}$ response and the risk of edema with MFN and SUs (Table 2).

\section{Fracture}

With TZDs, we found no evidence for an association between $\mathrm{HbA1c}$ response and the risk of a fracture (Figure 2D). There was also no evidence of an association with MFN and SUs (Tables 2).

\section{Associations using standard time-to- event approaches}

Results using the LOCF approach (Model 3: LOCF) were generally consistent with those from the current value joint models (Model 1: JMcv) (Table 2 and Figure 2). The exception was for TZDs and edema, for which, in contrast to the joint model, we found no evidence of an association using the LOCF model. Using Model 4: $6 \mathrm{mR}$ (where $\mathrm{HbA} 1 \mathrm{c}$ response was estimated from a single 6 month value), we found no evidence of any association between 
Table 2 HRs for the association between $\mathrm{HbAlc}$ response and risk of side effects (models $\mathrm{I}-3$ )

\begin{tabular}{|c|c|c|c|}
\hline Side effect & Model I: JMcv & Model 2: JMcum & Model 3: LOCF \\
\hline \multicolumn{4}{|l|}{ MFN } \\
\hline $\mathrm{Gl}$ & $0.82(0.67,1.01), P=0.06$ & $0.90(0.8 \mathrm{I}, \mathrm{I} .00), P=0.06$ & $0.85(0.74,0.96), P=0.01$ \\
\hline Hypoglycemia & $1.01(0.63,1.62), P=0.96$ & $1.22(0.93,1.60), P=0.15$ & I. $19(0.88, I .60), P=0.25$ \\
\hline Edema & $1.16(0.70,1.92), P=0.58$ & $1.09(0.88, I .36), P=0.42$ & $1.07(0.74, I .56), P=0.71$ \\
\hline Fracture & $0.83(0.48, I .44), P=0.5 \mathrm{I}$ & $\mathrm{I} .00(0.78, \mathrm{I} .27), P=0.98$ & $0.98(0.69,1.39), P=0.92$ \\
\hline \multicolumn{4}{|l|}{ SU } \\
\hline $\mathrm{Gl}$ & $0.88(0.69, I . I I), P=0.28$ & $1.03(0.92, I .17), P=0.58$ & $0.90(0.77,1.05), P=0.19$ \\
\hline Hypoglycemia & $1.41(1.04,1.91), P=0.03$ & $1.09(0.93, I .29), P=0.28$ & $1.4 \mathrm{I}(\mathrm{I} .12, \mathrm{I} .77), P=0.003$ \\
\hline Edema & $1.31(0.85,2.02), P=0.23$ & $1.09(0.87, I .36), P=0.45$ & $0.87(0.67, I .13), P=0.28$ \\
\hline Fracture & $1.16(0.70,1.92), P=0.58$ & $1.09(0.88, I .36), P=0.42$ & $\mathrm{I} .00(0.64, \mathrm{I} .58), P=0.68$ \\
\hline \multicolumn{4}{|l|}{ TZD } \\
\hline $\mathrm{GI}$ & $1.21(0.94, I .55), P=0.13$ & $\mathrm{I} .05(0.93, \mathrm{I} . \mathrm{I}), P=0.44$ & $\mathrm{I} .04(0.87, \mathrm{I} .26), P=0.65$ \\
\hline Hypoglycemia & $1.98(1.25,3.15), P=0.004$ & $1.37(\mathrm{I} .1 \mathrm{I}, \mathrm{I} .7), P=0.003$ & $\mathrm{I} .44(0.98,2.12), P=0.07$ \\
\hline Edema & $1.45(1.05,2.01), P=0.03$ & $1.22(1.07,1.38), P=0.003$ & I.0I $(0.80,1.27), P=0.94$ \\
\hline Fracture & $1.10(0.72, I .68), P=0.65$ & $1.09(0.93,1.29), P=0.28$ & $\mathrm{I} .05(0.72, \mathrm{I} .52), P=0.8 \mathrm{I}$ \\
\hline
\end{tabular}

Notes: HRs $(95 \% \mathrm{Cl})$ represent the increase in risk of a side effect for a $1 \%$ greater absolute $\mathrm{HbAlc}$ response. A HR of greater than I indicates an increased risk of a side effect with greater $\mathrm{HbAlc}$ response.

Abbreviations: Gl, gastrointestinal; JMcum, joint model cumulative HbAlc; JMcv, joint model current value; LOCF, last observation carried forward; MFN, metformin; SU, sulfonylurea; TZD, thiazolidinedione.

HbAlc response and the risk of side effects except for GI events with MFN (HR per 1\% absolute increase in 6-month HbA1c response, 0.74 [95\% CI 0.60, 0.91], Table S5).

\section{Discussion}

Our study shows that joint modeling can be a useful approach for evaluating associations between the benefits and risks of drug therapy. Using joint models for longitudinal and timeto-event data, we were able to show important differences in the associations between drug response and the risk of established side effects for three widely used type 2 diabetes drugs. We also found differences in the association between each of current and cumulative drug response and the risk of side-effects, suggesting underlying differences in the nature of associations for different drugs. Our results have implications for any precision medicine approach to type 2 diabetes therapy. More generally, they highlight the potential for the widespread application of joint longitudinal-survival modeling to evaluate the benefits and risks of both new and established medications.

\section{Advantages and disadvantages of joint models to evaluate the association between drug response and risk of side effects}

We found a key advantage of joint models to be their flexibility. Different specifications of the joint model gave important additional insight into the underlying nature of associations between HbA1c response and side effects. These insights fitted with what is known about the pharmacological action of the different drugs. Current, but not cumulative, HbAlc response was associated with an increased risk of hypoglycemia with SUs. This is expected as hypoglycemia is a side effect related to short-term fluctuations in blood glucose, rather than long-term exposure. In contrast, for edema with TZDs, which is less likely to relate to short-term fluctuations in blood glucose, we observed associations for both current and cumulative $\mathrm{HbA} 1 \mathrm{c}$ responses.

We also found associations with joint models that were missed by simpler approaches. With edema with TZD therapy, there was no association using the LOCF approach but a clear association using both specifications of the joint model. This is likely due to the reduced bias and increased efficiency of the joint model compared with the LOCF approach, which does not correct for measurement error in the longitudinal HbAlc response. ${ }^{5,7}$ In general, HRs using the LOCF approach had the same direction of association but were attenuated compared with those obtained from the current value joint model, in keeping with previous comparisons. ${ }^{4,17}$ We found that a single measure of HbA1c at 6 months was insufficient to show the evidence of an association between $\mathrm{HbA} 1 \mathrm{c}$ response and side effects, with the exception of GI side effects with MFN where the association was consistent with the joint model.

There are some settings where joint models may be more limited. ADOPT was a large randomized, double-blinded trial, and in this dataset, we found joint models to be useful to evaluate the association between response and relatively common side effects. Increasingly, similar trial datasets are 
available for researchers to address secondary research questions. ${ }^{13,18}$ It may be more challenging to apply joint modeling in other datasets. In particular, the potential of recording bias should be considered if conducting similar studies in electronic health records, although greater sample size may offer the opportunity to study rarer side effects. Testing the specificity of results to drugs known to cause the side effect by comparison with "negative control" drugs may be a useful starting point. Joint models may also be harder to apply to study associations between drug response and acute or allergic side effects that occur immediately after starting therapy. This was apparent in our analysis, as although we included over 1,000 participants for each drug, participants who developed an early side effect prior to a first on-therapy HbA1c were excluded, and this is a particular limitation of our analysis of hypoglycemia with SUs. Another limitation of the joint modeling framework applied in this study is the assumption of a fixed association between longitudinal $\mathrm{HbA1c}$ and the risk of each side effect. While inspection of residual plots indicated that this was an appropriate strategy, it is certainly plausible that associations could change with therapy duration, and incorporating duration of therapy as a time-varying effect within the joint modeling framework would be of considerable interest. Similarly, an extension of the joint modeling framework to robustly incorporate drug dose could yield further insight to complement the response:side effect associations evaluated in this study. Evaluating the impact of dose is a particular challenge in trials of drug efficacy such as ADOPT, as participants could be both uptitrated based on reaching glycemic thresholds and downtitrated if a randomized medication was poorly tolerated.

\section{Implications for a precision medicine approach to type 2 diabetes therapy}

Our findings for the different drugs have implications for any future precision medicine approach to type 2 diabetes therapy. Greater MFN drug response was not associated with an increased risk of GI side effects, and this suggests great potential to target therapy if patients likely to have greater drug response can be robustly identified. ${ }^{19}$ However, targeting SUs and TZDs to patients may be difficult as good responders are likely to be at increased risk of, respectively, hypoglycemia and edema. Our findings highlight the vital importance of considering both differential drug response and the risk of side effects in precision medicine studies, and this has been overlooked in previous work..$^{20,21}$

Our findings do not however preclude a precision medicine approach for SUs and TZDs. Identification of charac- teristics associated with either, but not both, improved drug response or lower risk of side effects may allow the targeting of these therapies. Furthermore, decisions on therapy should ultimately be informed by absolute rather than relative risks of benefit or harm. ${ }^{1}$ For example, if patients likely to respond well to a TZD can be identified, then, a TZD may still be an appropriate option for patients whose absolute risk of developing a side effect is sufficiently low.

\section{Comparison with other studies}

To our knowledge, this is the first evaluation of the association between $\mathrm{HbA} 1 \mathrm{c}$ response and the risk of side effects for any of the three drugs, except for hypoglycemia with SUs. Our results for SUs are consistent with previous observational studies that have examined the association between hypoglycemia and achieved on-therapy HbA1c (rather than $\mathrm{HbA} 1 \mathrm{c}$ response). ${ }^{22,23}$ In the ACCORD trial, participants with the greatest $\mathrm{HbA} 1 \mathrm{c}$ response at 4 months had a reduced rather than increased risk of hypoglycemia, although this can be explained by the fact that, in ACCORD, the participants with least initial response were more likely to be on insulin, the therapy with by far the strongest association with hypoglycemia. ${ }^{24}$

In this study, we found an unexpected association between greater response to TZD therapy and an increased risk of hypoglycemia, but no evidence of an association with MFN response, which would have indicated a positive association between increased drug response and increased risk of hypoglycemia was a more general characteristic of glucoselowering therapy. This is an interesting finding for which there is no clear biological explanation, and it would be of interest to examine whether the association can be replicated in other datasets. The association between edema and $\mathrm{HbA} 1 \mathrm{c}$ response with TZDs is not unexpected as the mechanisms underlying both glucose-lowering and fluid retention are thought to relate to Peroxisome proliferator-activated receptor gamma (PPAR- $\boldsymbol{\gamma}$ ) stimulation. ${ }^{25}$ With MFN, there is no clear biological reason for the association between greater $\mathrm{HbA} 1 \mathrm{c}$ response and a lower risk of GI events. One possible explanation is decreased drug adherence in patients experiencing mild GI symptoms prior to the event being recorded.

\section{Future work}

There is great potential to apply joint modeling to evaluate the association between drug response and the risk of side effects for the other drug options in type 2 diabetes and to study drug therapy in other diseases. Our findings also suggest a potential application of joint modeling as an efficient tool for understanding the risk-benefit trade-off at the individual level in 
drug development. ${ }^{26}$ For precision medicine, the joint models used in this study could be extended to explore clinical features and biomarkers associated with drug response, the risk of side effects, or both. ${ }^{27,28}$ Alternative model specifications, such as evaluation of the effect of HbAlc response slope, ${ }^{6}$ the weighting of cumulative HbAlc effects by recency, ${ }^{15}$ the incorporation of multiple longitudinal biomarkers, ${ }^{29}$ and exploration of time-varying drug effects, may provide further insight into the nature of associations between response and side effects. Similarly, incorporation of robust dose adjustment within the joint modeling framework, for example, testing weighted cumulative drug associations, ${ }^{30,31}$ could allow much greater understanding of the impact of different levels of drug exposure on both response and adverse events. Many of these are areas of current methodological development; a general mathematical presentation of joint modeling for simultaneously evaluating risks and benefits of medication would be a useful next step.

\section{Conclusion}

Joint modeling is a useful and efficient method to evaluate associations between continuous drug response and time to side effects. Our study suggests the potential for the application of joint modeling in both drug development and precision medicine research to evaluate the benefits and risks of medications. In type 2 diabetes, any future precision approach to SU and TZD therapy should consider the likely increased risk of, respectively, hypoglycemia and edema, if targeting these therapies at patients likely to have the greatest drug response.

\section{Abbreviations}

ADOPT, A Diabetes Outcome Progressing Trial; GSK, GlaxoSmithKline; MFN, metformin; SU, sulfonylurea; TZD, thiazolidinedione

\section{Data statement}

No additional data are available from the authors, although the individual participant data from the ADOPT trial used in this study are available from GlaxoSmithKline on application via www.clinicalstudydatarequest.com.

\section{Acknowledgment}

This work was supported by the Medical Research Council (UK) (Grant MR/N00633X/1).

\section{Author contributions}

JMD and WEH led the data analysis. All authors had full access to all of the data and take responsibility for the integrity of the data and the accuracy of the data analysis. WEH is the guarantor. All authors contributed to the study design, data analysis, drafting and revising the article, gave final approval of the version to be published, and agree to be accountable for all aspects of the work.

\section{Disclosure}

ATH is a NIHR Senior Investigator and a Wellcome Trust Senior Investigator. ERP is a Wellcome Trust New Investigator (102820/Z/13/Z). AGJ is supported by an NIHR Clinician Scientist award. ATH and BMS are supported by the NIHR Exeter Clinical Research Facility. WEH received additional support from IQVIA and the National Institute for Health Research (NIHR) Collaboration for Leadership in Applied Health Research and Care South West Peninsula (NIHR CLAHRC South West Peninsula). The views expressed are those of the authors and not necessarily those of the NHS, the NIHR, or the Department of Health and Social Care. The funders had no role in any part of the study or in any decision about publication. WEH receives a grant from IQVIA, and ERP receives personal fees from Lily, Novo Nordisk, and Astra Zeneca. The authors report no other conflicts of interest in this work.

\section{References}

1. Hingorani AD, Windt DA, Riley RD, et al; PROGRESS Group. Prognosis research strategy (PROGRESS) 4: stratified medicine research. BMJ. 2013;346:e5793.

2. Dahabreh IJ, Hayward R, Kent DM. Using group data to treat individuals: understanding heterogeneous treatment effects in the age of precision medicine and patient-centred evidence. Int J Epidemiol. 2016;45(6):2184-2193.

3. Henderson R, Diggle P, Dobson A. Joint modelling of longitudinal measurements and event time data. Biostatistics. 2000;1(4):4 65-480.

4. Asar Ö, Ritchie J, Kalra PA, Diggle PJ. Joint modelling of repeated measurement and time-to-event data: an introductory tutorial. Int $J$ Epidemiol. 2015;44(1):334-344.

5. Ibrahim JG, Chu H, Chen LM. Basic concepts and methods for joint models of longitudinal and survival data. J Clin Oncol. 2010;28(16):2796-2801.

6. Rizopoulos D. Joint Models for Longitudinal and Time-To-Event Data: with Applications in R. Boca Raton: Chapman and Hall/CRC; 2012.

7. Lawrence Gould A, Boye ME, Crowther MJ, et al. Joint modeling of survival and longitudinal non-survival data: current methods and issues. Report of the DIA Bayesian joint modeling working group. Stat Med. 2015;34(14):2181-2195.

8. Köhler M, Beyerlein A, Vehik K, et al; TEDDY study group. Joint modeling of longitudinal autoantibody patterns and progression to type 1 diabetes: results from the TEDDY study. Acta Diabetol. 2017;54(11):1009-1017.

9. Fournier MC, FoucherY, Blanche P, Buron F, Giral M, Dantan E. A joint model for longitudinal and time-to-event data to better assess the specific role of donor and recipient factors on long-term kidney transplantation outcomes. Eur J Epidemiol. 2016;31(5):469-479.

10. Andersson TM, Crowther MJ, Czene K, Hall P, Humphreys K. Mammographic density reduction as a prognostic marker for postmenopausal breast cancer: results using a joint longitudinal-survival modeling approach. Am J Epidemiol. 2017;186(9):1065-1073. 
11. Tsiatis AA, Degruttola V, Wulfsohn MS. Modeling the relationship of survival to longitudinal data measured with error. applications to survival and CD4 counts in patients with AIDS. JAm Stat Assoc. 1995;90(429):27-37.

12. Kahn SE, Haffner SM, Heise MA, et al; ADOPT Study Group. Glycemic durability of rosiglitazone, metformin, or glyburide monotherapy. $N$ Engl J Med. 2006;355(23):2427-2443.

13. Clinical Study Data Request [homepage on the Internet]. Available from: https://clinicalstudydatarequest.com/. Accessed February 15, 2018.

14. Jones AG, Lonergan M, Henley WE, et al. Should studies of diabetes treatment stratification correct for baseline HbA1c? PLoS One. 2016;11(4):e0152428.

15. Mauff K, Steyerberg EW, Nijpels G, van der Heijden A, Rizopoulos D. Extension of the association structure in joint models to include weighted cumulative effects. Stat Med. 2017;36(23):3746-3759.

16. Rizopoulos D. JM: An $R$ package for the joint modelling of longitudinal and time-to-event data. J Stat Softw. 2010;35(9):1-33.

17. Sweeting MJ, Thompson SG. Joint modelling of longitudinal and timeto-event data with application to predicting abdominal aortic aneurysm growth and rupture. Biom J. 2011;53(5):750-763.

18. The YODA Project [homepage on the Internet]. Available from: http:// yoda.yale.edu/. Accessed February 15, 2018.

19. GoDARTS and UKPDS Diabetes Pharmacogenetics Study Group, Wellcome Trust Case Control Consortium 2, Zhou K, et al. Common variants near ATM are associated with glycemic response to metformin in type 2 diabetes. Nat Genet. 2011;43(2):117-120.

20. Dennis JM, Shields BM, Hill AV, et al; MASTERMIND Consortium. Precision medicine in type 2 diabetes: clinical markers of insulin resistance are associated with altered short- and long-term glycemic response to DPP-4 inhibitor therapy. Diabetes Care. 2018;41(4):705-712.

21. Jones AG, McDonald TJ, Shields BM, et al; PRIBA Study Group Markers of $\beta$-cell failure predict poor glycemic response to GLP-1 receptor agonist therapy in type 2 diabetes. Diabetes Care. 2016;39(2): $250-257$.
22. Lipska KJ, Warton EM, Huang ES, et al. HbA1c and risk of severe hypoglycemia in type 2 diabetes: the Diabetes and Aging Study. Diabetes Care. 2013;36(11):3535-3542.

23. Yu S, Fu AZ, Engel SS, Shankar RR, Radican L. Association between hypoglycemia risk and hemoglobin A1C in patients with type 2 diabetes mellitus. Curr Med Res Opin. 2016;32(8):1409-1416.

24. Miller ME, Bonds DE, Gerstein HC, et al; ACCORD Investigators. The effects of baseline characteristics, glycaemia treatment approach, and glycated haemoglobin concentration on the risk of severe hypoglycaemia: post hoc epidemiological analysis of the ACCORD study. BMJ. 2010;340:b5444.

25. Davidson MA, Mattison DR, Azoulay L, Krewski D. Thiazolidinedione drugs in the treatment of type 2 diabetes mellitus: past, present and future. Crit Rev Toxicol. 2018;48(1):52-108.

26. Costa MJ, Drury T. Bayesian joint modelling of benefit and risk in drug development. Pharm Stat. 2018;17(3):248-263.

27. Hattersley AT, Patel KA. Precision diabetes: learning from monogenic diabetes. Diabetologia. 2017;60(5):769-777.

28. Dennis JM, Henley WE, Weedon MN, et al. Sex and BMI; MASTERMIND Consortium Alter the benefits and risks of sulfonylureas and thiazolidinediones in type 2 diabetes: a framework for evaluating stratification using routine clinical and individual trial data. Diabetes Care. 2018;41(9):1844-1853.

29. Hickey GL, Philipson P, Jorgensen A, Kolamunnage-Dona R. Joint modelling of time-to-event and multivariate longitudinal outcomes: recent developments and issues. BMC Med Res Methodol. 2016;16(1):117.

30. Abrahamowicz M, Beauchamp ME, Sylvestre MP. Comparison of alternative models for linking drug exposure with adverse effects. Stat Med. 2012;31(11-12):1014-1030.

31. Abrahamowicz M, Bartlett G, Tamblyn R, du Berger R. Modeling cumulative dose and exposure duration provided insights regarding the associations between benzodiazepines and injuries. J Clin Epidemiol. 2006;59(4):393-403.
Clinical Epidemiology

\section{Publish your work in this journal}

Clinical Epidemiology is an international, peer-reviewed, open access, online journal focusing on disease and drug epidemiology, identification of risk factors and screening procedures to develop optimal preventative initiatives and programs. Specific topics include: diagnosis, prognosis, treatment, screening, prevention, risk factor modification,

Submit your manuscript here: https://www.dovepress.com/clinical-epidemiology-journal

\section{Dovepress}

systematic reviews, risk and safety of medical interventions, epidemiology and biostatistical methods, and evaluation of guidelines, translational medicine, health policies and economic evaluations. The manuscript management system is completely online and includes a very quick and fair peer-review system, which is all easy to use. 